\title{
Electron Transport via Local Polarons at Interface Atoms
}

\author{
M. Berthe, ${ }^{1}$ A. Urbieta, ${ }^{1}$ L. Perdigão, ${ }^{1}$ B. Grandidier, ${ }^{1}$ D. Deresmes,,${ }^{1}$ C. Delerue,${ }^{1}$ D. Stiévenard,${ }^{1}$ R. Rurali, ${ }^{2}$ \\ N. Lorente, ${ }^{2, *}$ L. Magaud, ${ }^{3}$ and P. Ordejón ${ }^{4}$ \\ ${ }^{1}$ Institut d'Electronique, de Microélectronique, et de Nanotechnologie, IEMN (CNRS, UMR 8520), Département ISEN, \\ 41 bd Vauban, 59046 Lille Cédex, France \\ ${ }^{2}$ LCAR (UMR 5589), Université Paul Sabatier, 118 route de Narbonne, 31062 Toulouse, Cédex France \\ ${ }^{3}$ Laboratoire d'Etude des Propriétés Electroniques des Solides (LEPES-CNRS), BP 166, 38042 Grenoble Cédex 9, France \\ ${ }^{4}$ Institut de Ciència de Materials de Barcelona CSIC, 08193, Barcelona, Spain
}

(Received 17 March 2006; published 13 November 2006)

\begin{abstract}
Electronic transport is profoundly modified in the presence of strong electron-vibration coupling. We show that in certain situations, the electron flow takes place only when vibrations are excited. By controlling the segregation of boron in semiconducting $\operatorname{Si}(111)-\sqrt{3} \times \sqrt{3} R 30^{\circ}$ surfaces, we create a type of adatom with a dangling-bond state that is electronically decoupled from any other electronic state. However, probing this state with scanning tunnelling microscopy at $5 \mathrm{~K}$ yields high currents. These findings are rationalized by ab-initio calculations that show the formation of a local polaron in the transport process.
\end{abstract}

DOI: 10.1103/PhysRevLett.97.206801

Electronic transport through atomic systems determines the way that devices behave on the atomic scale. This has important consequences in the development of molecular electronics [1]. Hence, the field of electronic transport on the atom scale is subject to great recent activity $[1,2]$. These efforts have permitted to understand how electronic current flows according to the geometry and the nature of the atomic components of the studied systems. Landauer theory has proven to be of great value in the rationalization of the many careful experiments addressing transport through a few systems [2]. However, this type of understanding needs to be revisited when vibrations affect electron transport.

The role of vibrations in electronic transport is thus attracting a lot of interest. Indeed, recent theoretical work [3] has shown that the coupling between electrons and vibrations can efficiently suppress the electron flow leading to a Franck-Condon blockade regime. This transport regime can have tremendous impact in the device's shot noise and the avalanche-like type of electron flow [4]. Interesting physics has been explored when combining electron-electron and electron-vibration interactions $[5,6]$. Regarding the electron-vibration strong-coupling regime, great theoretical interest has been devoted to the description of polarons as electronic excitations coupled with atomic vibrations $[7,8]$. Recently, using the lowestorder expansion (LOE) on the electron-vibration coupling, simulated inelastic electron tunnelling spectra have been successfully compared with experimental work [9]. Also at the ab-initio level, a detailed theory-experiment comparison within the LOE has been undertaken for a group of hydrocarbon molecules connected to gold electrodes [10]. A first all-order calculation at the ab-initio level [11] has permitted to gain insight in the intricacies of vibration excitation in ballistic conductance experiments [12]. Yet,
PACS numbers: 73.43.Jn, 63.22.+m, 68.37.Ef, 73.63.-b

it is desirable to extend these studies to the strong-coupling limit where the above effects may take place.

Unfortunately, there are few conduction experiments dealing with the physical regimes explored in the limit of strong electron-vibration coupling. Wu and coworkers [13] have been able to explore the region of medium electronvibration coupling by going into the limit of very weak molecule-electrode couplings. They show the dramatic signature of vibrations in electronic transport. To our knowledge, there is one only transport experiment where the regime of strong electron-vibration coupling has been attained. Repp et al. [14] give experimental evidence that the conductance dependence with voltage is modified by the electron-vibration interactions in such a way that the effect of the electronic structure behind the conductance behavior is not directly identifiable.

The present work addresses the physics treated in the above references [3-6] by providing electronic transport measurements in the presence of strong electron-vibration coupling in the basically ideal case of transport through a single atomic state, decoupled from other electronic states. The measurements are performed by using a scanning tunnelling microscope (STM) tip as an electrode and a passivated boron-doped $\mathrm{Si}(111)-\sqrt{3} \times \sqrt{3} R 30^{\circ}$ surface as the other electrode at $5 \mathrm{~K}$. When a $\mathrm{Si}$ adatom is not passivated by the subsurface boron (B) layer, its electronic structure corresponds to a dangling-bond (DB) state energetically localized in the energy gap of the B-doped $\mathrm{Si}(111)-\sqrt{3} \times \sqrt{3} R 30^{\circ}$ surface. Hence, the electron current should be zero until the STM tip enters in mechanical contact with the adatom. Surprisingly, far before contact, the current easily reaches $1 \mathrm{nA}$. This is possible because of the large electron-vibration coupling on the adatom, leading to the formation of a local polaron. In the present case, not only does the electron-vibration coupling modify the 
conductance spectrum, but it is strictly required to establish the current.

When a B-doped $\mathrm{Si}(111)$ crystal is heated, B dopants diffuse to the surface and replace $\mathrm{Si}$ atoms just under the adatom position. This process leads to charge transfer from the $\mathrm{Si}$ adatoms to the substitutional $\mathrm{B}$ atoms, making the adatom's DB state passivated [15]. By controlling the heating of the Si crystal, the number of substitional B atoms can be tuned, so that only a few Si DB, bearing a localized state, are left.

Figure 1(a) shows a constant-current STM image of a B-doped $\operatorname{Si}(111)$ surface with the characteristic $\sqrt{3} \times$ $\sqrt{3} R 30^{\circ}$ reconstruction. The measurements have been performed at a temperature of $5 \mathrm{~K}$. Most of the $\mathrm{Si}$ adatoms appear dark, consistent with the passivation of the DB states [15]. However, two isolated bright adatoms can be seen. Their corresponding DB wave functions are localized into the region defined by the six first surface neighbors of the adatom, giving rise to a bright flowerlike shape.

Superimposed to the contrast of the passivated DBs, faint dark depressions are observed in Fig. 1(a). They turn into protrusions when flipping the voltage sign to image filled states, Fig. 1(b). These features are the signature of subsurface dopant atoms [16,17]. They result from the local screened Coulomb potential-induced band bending surrounding the charged B impurities. Such impurities are lying at least four layers below the surface plane. Since in these four layers B impurities are located in symmetrical positions with respect to the adatoms, analyzing the corrugation profile across the protrusion allows the counting of the impurities in each layer [18]. Below the sixth layer, we estimate a concentration of $5.1 \times$

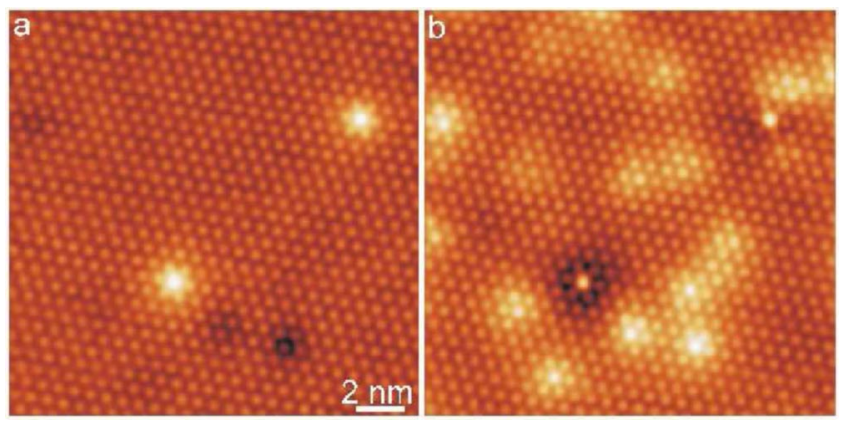

FIG. 1 (color online). Visualization of passivated and isolated $\mathrm{Si}$ dangling bonds on the $\mathrm{Si}(111) \sqrt{3} \times \sqrt{3} R 30^{\circ}$-B surface observed at 5 K. (a) Empty state STM image. The majority of the adatoms appears dark (adatoms with B atoms underneath). Unlike these adatoms, two $\mathrm{Si}$ adatoms appear much brighter and correspond to a configuration with a $\mathrm{Si}$ atom underneath. The height difference between the dark and bright adatoms is $0.6 \AA\left(V_{\text {sample }}=+1.8 \mathrm{~V}\right)$. (b) Filled state STM image of the same area $\left(V_{\text {sample }}=-0.4 \mathrm{~V}\right)$. The numerous bright protrusions, which show a more or less dark contrast in the empty state image, correspond to subsurface B dopants, located at least 4 atomic layers below the surface.
$10^{19} \mathrm{~cm}^{-3}$, thus permitting the formation of a degenerated band of acceptor states positioned at the top of the valence band.

To establish a direct link between the adatom contrast and their electronic nature, differential-conductance spectroscopy measurements were done on dark and bright adatoms, Fig. 2. The spectrum acquired above a dark adatom site presents the Fermi level $E_{F}$ positioned close to the top of the valence band, consistent with the formation of the just mentioned acceptor band. Two distinct features demonstrate that the dark Si DBs are electronically passivated: (i) the occurrence of a $1.3 \mathrm{eV}$ gap and (ii) the presence of a strong peak positioned $0.4 \mathrm{eV}$ above the bottom of the conduction band (CB) marked with an arrow, in agreement with the formation of an unoccupied band resonant with the conduction band due to charge transfer from Si to B [19]. In contrast, the spectrum on a bright adatom exhibits a distinct feature in the band gap region with respect to the dark adatom sites. A peak is now visible at an energy of $0.6 \mathrm{eV}$ and is related to the contribution to the current of the non resonant DB state of the bright adatom. Such a strong peak in the band gap explains the topographical contrast between the two types of $\mathrm{Si}$ adatoms in the empty state images of Fig. 1(a).

Surprisingly, the isolated DB state can hold an electron current bigger than $1 \mathrm{nA}$ at a temperature of $5 \mathrm{~K}$. This finding is at odds with the above picture of a DB state in the surface band gap. In order to explain the experimental large current through the DB state, several mechanisms can be

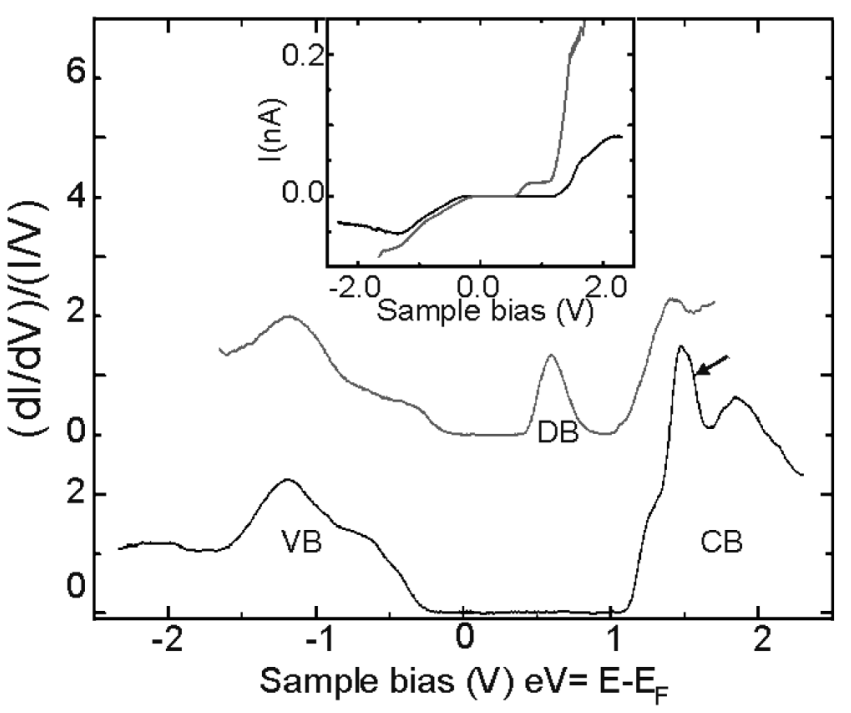

FIG. 2. Normalized conductivity spectra obtained on dark (black line) and bright ( gray line) adatom sites at a temperature of $5 \mathrm{~K}$. The peak related to the localized dangling-bond state, the conduction, and valence bands are, respectively, labeled $\mathrm{DB}, \mathrm{CB}$, and VB. The arrow points toward the peak related to the surface state band of the passivated dangling bonds. The tip's height is fixed at the feedback conditions (black line) $+2.3 \mathrm{~V}, 80 \mathrm{pA}$ and (gray line) $+1.7 \mathrm{~V}, 250 \mathrm{pA}$. 
invoked [20]: (i) hopping of the electron among the different DB states; however, this is not possible due to the small concentration of bright adatoms on the surface; (ii) thermal excitation of the electron towards the $\mathrm{CB}$ by phonon absorption; yet, this process can be ruled out at $5 \mathrm{~K}$; (iii) as a result, only processes involving the recombination of the electron with a hole from the acceptor band are possible if the excess electron energy is absorbed by either photon emission or atomic vibrations. Considering a hole concentration of $5 \times 10^{19} \mathrm{~cm}^{-3}$, a rough estimation of the recombination rate [21] for a radiative recombination yields $5 \mathrm{~ms}^{-1}$. This rate is however many orders of magnitude too small to account for the measured currents that are in the range of $10 \mathrm{pA}$ to $1 \mathrm{nA}$. Rather, the electron transfer takes place because the excess energy is given to local vibrations.

When a DB changes its charge state, the Si adatom is displaced [22,23]: its configuration coordinate, $Q$, varies from $Q=0$ to $Q=Q^{-}$, Fig. 3. Since the electron transfer is much faster than the adatom relaxation, the negatively charged adatom is left in a vibrationally excited state. Next, the electron is transferred to an empty state of the acceptor band to recombine non radiatively with a hole. Thus, the
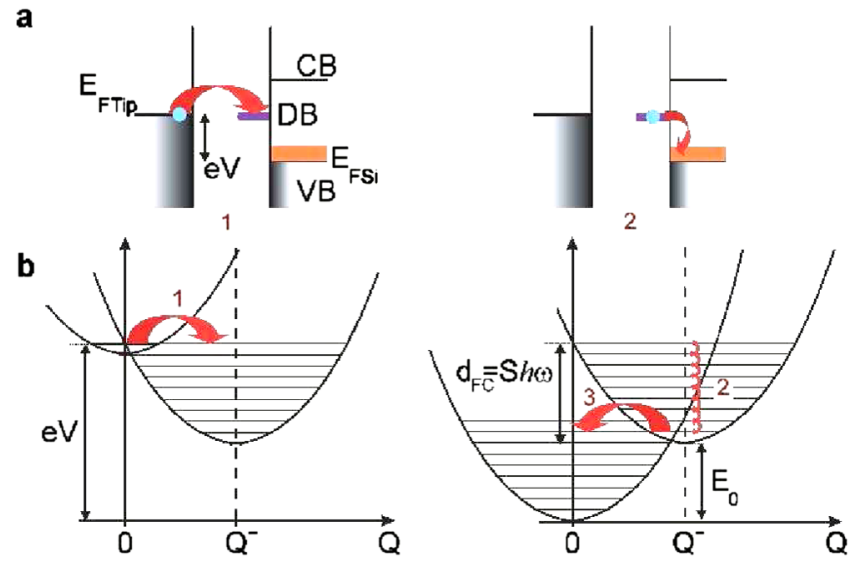

FIG. 3 (color online). (a) Energy diagrams sketching the twostep transport process: (1) the energy, eV, of the electron in the tip is tuned via the STM bias voltage, to become resonant with the dangling-bond state (DB) at a given vibrational state; (2) when sufficient energy has been transferred to the adatom, the probability of nonradiative transfer into the partially empty band of Boron impurities becomes high enough. The conduction and valence bands are, respectively, labeled by $\mathrm{CB}$ and VB, the tip and silicon Fermi levels by $E_{F T i p}$ and $E_{F S i}$. (b) Total energy curves as a function of the vibrational coordinate, $Q$, of the dangling-bond. On the left graph (process 1), the adatom in its ground state (top parabola) changes its charge state and its total energy corresponds to the bottom parabola, centered at $Q=Q^{-}$. On the second diagram, the adatom in the excited state (top parabola) may dissipate up to $S$ quanta of vibration of energy $\hbar \omega$, corresponding to the Franck-Condon shift $d_{\mathrm{FC}}$ (process 2). Finally, the electron is transferred into the degenerated band of Boron impurities, leaving the adatom in a vibrationally excited state about its initial position (process 3 ). adatom returns to its neutral oscillator state, centered at $Q=0$, in a highly excited vibrational state. Eventually, the vibrational damping of the adatom can change the intermediate vibrational state.

To prove this mechanism, we measured $d^{2} I / d V^{2}$ spectra on bright adatoms. Figure 4(a) shows $d I / d V$ and $d^{2} I / d V^{2}$ spectra acquired concurrently in the energy range of the peak labeled DB, in Fig. 2. In the $d^{2} I / d V^{2}$ curve, equidistant peaks are observed, suggesting the vibrational excitation of the adatom, when electrons tunnel into the DB state
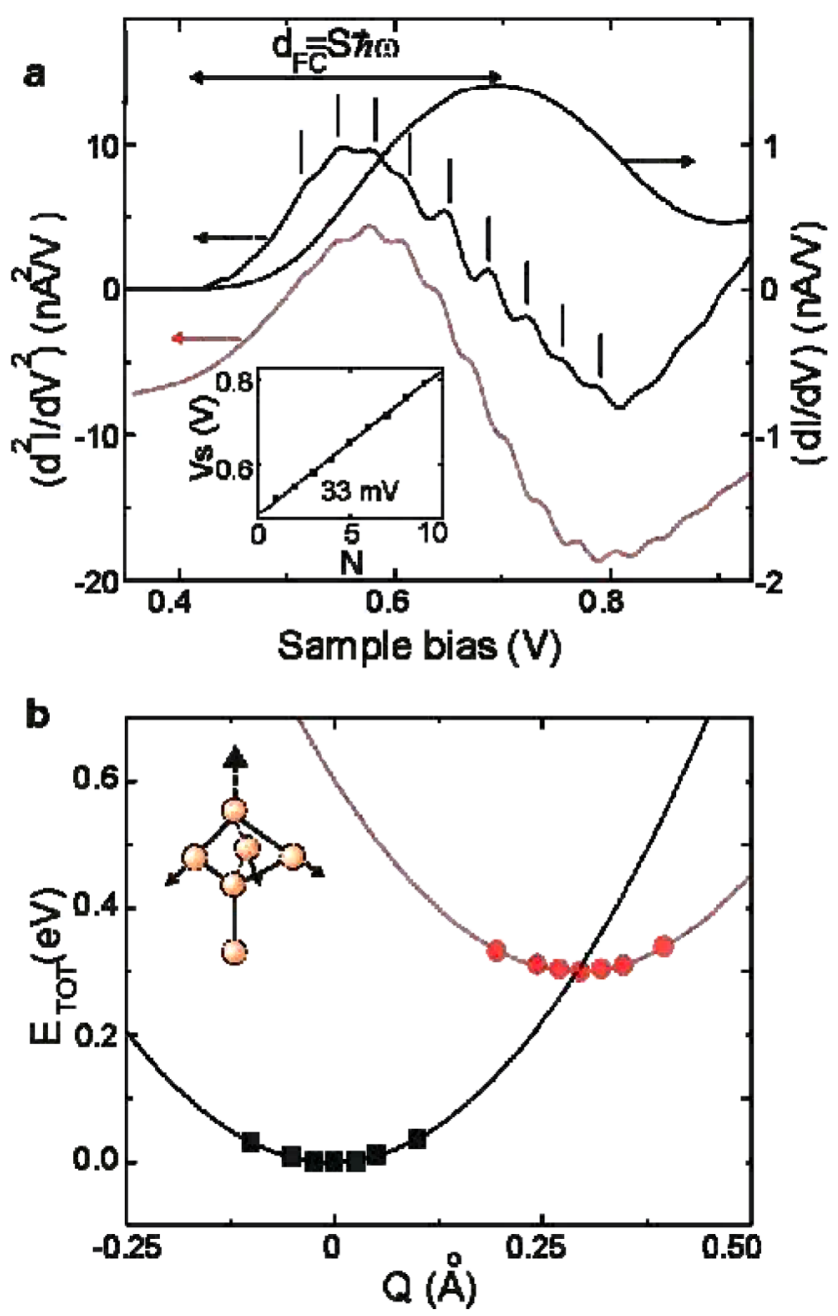

FIG. 4 (color online). (a) Experimental (upper) and calculated (lower) $d^{2} I / d V^{2}$ spectra, shifted for clarity and obtained on a bright adatom. The vertical lines indicate the position of equally spaced peaks in the spectrum. The Franck-Condon shift $d_{\mathrm{FC}}=$ $S \hbar \omega$ is deduced from the width at half maximum of the $d I / d V$ peak. The curves were acquired with feedback parameters $V_{\text {sample }}=+1.5 \mathrm{~V}$ and $I=500 \mathrm{pA}$ at $5 \mathrm{~K}$. Inset: Plot of the peak energy versus the peak number $N$ for the vibronic progression, yielding the energy of the vibrational mode. (b) Total energy of a neutral (lower) and a negative charge (upper) dangling-bond, calculated by density functional theory, versus the lattice coordinate $Q$ of the adatom (top atom in the atomic structure) for the stretching mode. 
[13]. From measurements obtained on different bright adatoms, the peak spacing is found to be $31 \pm 3 \mathrm{meV}$.

First-principles calculations [24] were used to determine both the electronic and vibrational structures of the bright adatoms that enter the simulation of the vibronic tunnelling current based on the tunnelling of an electron through a single site coupled to vibrations $[25,26]$. The calculations are performed for a DB state with and without an extra electron. The Huang-Rhys factor, $S$, is evaluated as the linear term of the harmonic fit to the total energy as a function of the configuration coordinate, $Q$, Fig. 4(b). Among all the local vibrational modes studied, only the stretching mode gives a strong electron-vibration coupling where $S=9.6$. For this mode, a quantum of vibration of $\hbar \omega=32.5 \mathrm{meV}$ is found. The lifetime of the electron in the dangling-bond is derived from Ref. [27]. The capture rate $W=1 / \tau$ is written as $W=p c_{0} R\left(T, E_{0}\right)$. The hole concentration $p$ is obtained from the observed concentration of subsurface dopant atoms. We assume the capture coefficient $c_{0}$ to be close to its value for the $\mathrm{Pb}$ defect [28], i.e., the dangling-bond defect at the $\mathrm{Si}-\mathrm{SiO}_{2}$ interface. Finally, $R\left(T, E_{0}\right)$ is a dimensionless function which is explicitly given in Ref. [28] and depends on the temperature $T$ and the ionization energy, $E_{0}$, of the DB. Since the peak related to the DB state is positioned at an energy of $0.7 \mathrm{eV}$, and this energy corresponds to the sum of the Frank-Condon shift $d_{\mathrm{FC}}=S \hbar \omega$ plus the ionization energy $E_{0}$, we deduce $E_{0}$ to be $0.4 \mathrm{eV}$. An estimation of the electron lifetime in the DB yields a lifetime of the order of $10^{-12} \mathrm{~s}$ at $5 \mathrm{~K}$ leading to currents of $1 \mathrm{nA}$. Taking into account these values, the calculation of the $d^{2} I / d V^{2}$ spectrum was performed, with excellent agreement with experiment, supporting the assignment of the peaks in Fig. 4(a) to vibronic states.

In summary, by using a scanning tunnelling microscope (STM) tip as an electrode and a passivated B-doped $\operatorname{Si}(111) \sqrt{3} \times \sqrt{3} R 30^{\circ}$ surface as the other electrode at $5 \mathrm{~K}$, the conductance of an unpassivated $\mathrm{Si}$ adatom was measured. The electronic structure of this adatom corresponds to a DB state energetically localized in the energy gap of the surface. Hence, this state is electronically decoupled from any other electronic state. Yet, we found large currents on the adatom DB that can be rationalized in a two-step picture: a tunnelling of the electron into the DB state and then its nonradiative recombination with a hole from the boron band. Our main finding is that the latter process is possible only if the adatom can absorb the electron excess energy by becoming vibrationally excited. This process is efficient enough only in the presence of a large electron-vibration coupling, giving rise to a polaronic-like conduction.

This work was supported by the European Community's Human Potential Programme under contract No. HPRNCT-2001-00320, NANOSPECTRA, by DGES-Spain's No. BFM2003-03372-C03, by the Generalitat de
Catalunya (No. 2005 SGR 683) and by the FrenchSpanish bilateral programme PICASSO. R. R. acknowledges the financial support of the Generalitat de Catalunya (NANOTEC). Computational resources at the Centre Informatique National de l'Enseignement Supérieur and the Centre de Calcul Midi-Pyrénées are gratefully acknowledged.

*Corresponding author: Electronic mail: lorente@ irsamc.ups-tlse.fr

[1] A. Nitzan and M. A. Ratner, Science 300, 1384 (2003).

[2] N. Agraït, A. Levy Yeyati, and J. M. van Ruitenbeek, Phys. Rep. 377, 81 (2003), and references therein.

[3] J. Koch and F. von Oppen, Phys. Rev. Lett. 94, 206804 (2005).

[4] J. Koch, M. E. Raikh, and F. von Oppen, Phys. Rev. Lett. 95, 056801 (2005); Phys. Rev. Lett. 96, 056803 (2006).

[5] P. S. Cornaglia, H. Ness, and D. R. Grempel, Phys. Rev. Lett. 93, 147201 (2004).

[6] Jens Paaske and Karsten Flensberg, Phys. Rev. Lett. 94, 176801 (2005).

[7] W. Koller, A. C. Hewson, and D. M. Edwards, Phys. Rev. Lett. 95, 256401 (2005).

[8] H. Ness and A. J. Fisher, Phys. Rev. Lett. 83, 452 (1999).

[9] M.-L. Bocquet, H. Lesnard, and N. Lorente, Phys. Rev. Lett. 96, 096101 (2006).

[10] M. Paulsson, T. Frederiksen, and M. Brandbyge, Nano Lett. 6, 258 (2006); Phys. Rev. B 72, 201101(R) (2005).

[11] T. Frederiksen et al., Phys. Rev. Lett. 93, 256601 (2004).

[12] N. Agraït et al., Phys. Rev. Lett. 88, 216803 (2002).

[13] S. Wu et al., Phys. Rev. Lett. 93, 236802 (2004).

[14] J. Repp et al., Phys. Rev. Lett. 95, 225503 (2005).

[15] I.-W. Lyo, E. Kaxiras, and Ph. Avouris, Phys. Rev. Lett. 63, 1261 (1989).

[16] P. Ebert, Surf. Sci. Rep. 33, 121 (1999).

[17] G. Mahieu et al., Phys. Rev. Lett. 94, 026407 (2005).

[18] R. M. Feenstra, J. M. Woodall, and G. D. Pettit, Phys. Rev. Lett. 71, 1176 (1993).

[19] T.M. Grehk, P. Mårtenson, and J.M. Nicholls, Phys. Rev. B 46, 2357 (1992); H. Q. Shi, M. W. Radny, and P. V. Smith, Phys. Rev. B 66, 085329 (2002).

[20] X. de la Broise et al., Phys. Rev. B 61, 2138 (2000).

[21] C. Delerue, G. Allan, and M. Lannoo, Phys. Rev. B 48, 11024 (1993).

[22] W. A. Harrison, Surf. Sci. 55, 1 (1976).

[23] M. Lannoo and P. Friedel, Atomic and Electronic Structure of Surfaces (Springer Verlag, New York, 1991).

[24] J. Soler et al., J. Phys. Condens. Matter 14, 2745 (2002); http://www.uam.es/siesta/.

[25] G. D. Mahan, Many-particles Physics (Plenum, New York, 2000).

[26] M. Lannoo and J. Bourgoin, Point Defects in Semiconductors (Springer Verlag, Berlin, 1983).

[27] C. Delerue, G. Allan, and M. Lannoo, Phys. Rev. B 48, 11024 (1993).

[28] D. Goguenheim and M. Lannoo, J. Appl. Phys. 68, 1059 (1990). 